\title{
Transcription initiation by human RNA polymerase II visualized at single-molecule resolution
}

\author{
Andrey Revyakin, ${ }^{1,5}$ Zhengjian Zhang, ${ }^{1,5}$ Robert A. Coleman, ${ }^{2}$ Yan Li, ${ }^{1}$ Carla Inouye, ${ }^{3}$ Julian K. Lucas, ${ }^{3}$ \\ Sang-Ryul Park, ${ }^{3}$ Steven Chu, ${ }^{4}$ and Robert Tjian ${ }^{1,3,6}$ \\ ${ }^{1}$ Janelia Farm Research Campus, Howard Hughes Medical Institute, Ashburn, Virginia 20147, USA; ${ }^{2}$ Albert Einstein College of \\ Medicine, Bronx, New York 10461, USA; ${ }^{3}$ QB3, Li Ka-shing Center, Department of Molecular and Cell Biology, University of \\ California at Berkeley, Berkeley, California 94720, USA; ${ }^{4}$ Formerly of University of California at Berkeley and Lawrence Berkeley \\ National Laboratory, Berkeley, California 94720, USA
}

Forty years of classical biochemical analysis have identified the molecular players involved in initiation of transcription by eukaryotic RNA polymerase II (Pol II) and largely assigned their functions. However, a dynamic picture of Pol II transcription initiation and an understanding of the mechanisms of its regulation have remained elusive due in part to inherent limitations of conventional ensemble biochemistry. Here we have begun to dissect promoter-specific transcription initiation directed by a reconstituted human Pol II system at singlemolecule resolution using fluorescence video-microscopy. We detected several stochastic rounds of human Pol II transcription from individual DNA templates, observed attenuation of transcription by promoter mutations, observed enhancement of transcription by activator Sp1, and correlated the transcription signals with real-time interactions of holo-TFIID molecules at individual DNA templates. This integrated single-molecule methodology should be applicable to studying other complex biological processes.

[Keywords: single-molecule fluorescence; Pol II transcription; preinitiation complex; reinitiation; unstructured probes; surface passivation]

Supplemental material is available for this article.

Received April 25, 2012; revised version accepted June 14, 2012.

RNA polymerase II (Pol II) is responsible for the flow of genetic information from DNA to messenger RNA (mRNA) in the eukaryotic cell. Our current mechanistic understanding of Pol II transcription is based on genetic studies (for review, see Hampsey 1998) and on biochemical fractionation of cell extracts (for review, see Orphanides et al. 1996; Roeder 1996). These studies have identified a cadre of general transcription factors (GTFs)-TFIIA, TFIIB, TFIID, TFIIE, TFIIF, and TFIIH - that, together with Pol II, assemble at the promoter into a so-called preinitiation complex (PIC) and direct accurate transcription initiation at a basal activity level. Further modulation of transcription activity depends on cis control elements in the DNA template that are recognized by sequence-specific activators/repressors assisted by a host of coactivators (Albright and Tjian 2000; Kornberg 2005). Although 40 years of research have successfully identified many of the key

\footnotetext{
${ }^{5}$ These authors contributed equally to this work.

${ }^{6}$ Corresponding author

E-mail jmlim@berkeley.edu

Article published online ahead of print. Article and publication date are online at http://www.genesdev.org/cgi/doi/10.1101/gad.194936.112.
}

players involved in Pol II transcription initiation and regulation, important questions dealing with dynamic aspects of the process remain unanswered due to population-averaging effects and limited time resolution of ensemble biochemical techniques.

Single-molecule techniques provide a means to directly monitor protein-nucleic acid and protein-protein interactions at subsecond time resolution, without the averaging effect of bulk biochemistry (Weiss 1999). For example, single-molecule techniques can address the order of PIC assembly and the timing of the release of individual GTFs following escape of Pol II from the promoter. Furthermore, single-molecule approaches can establish a direct correlation between GTF-GTF, activator-GTF, and GTF-DNA interactions and different transcription outcomes at individual DNA templates. Despite these capabilities, there has been a paucity of single-molecule methods tackling dynamic, multicomponent processes comparable in complexity to Pol II transcription initiation, with the exception of recent notable studies in protein translation and RNA splicing (Blanchard et al. 2004; Uemura et al. 2010; Hoskins et al. 2011). Here we developed a single-molecule methodology for direct visualization of transcription fac- 
tor-DNA interactions and detection of RNA production at the same individual DNA templates in a highly purified, fully reconstituted human Pol II system. These initial studies provide a single-molecule transcription platform that is responsive to core promoter elements, transcription inhibitors, and the prototypical sequence-specific DNAbinding activator Spl. We were able to directly count the number of transcripts produced at individual promoter DNA templates and correlate binding by the GTF TFIID-the component of the PIC that is primarily responsible for core promoter recognition-with transcriptional activity. The methodology described here opens new avenues toward a greater understanding of the dynamics of eukaryotic transcription and can be adapted to other complex multicomponent biological processes.

\section{Results}

\section{Rationale and method development}

We developed a custom wide-field total internal reflection fluorescence (TIRF) microscope to track the molecular events at individual DNA templates from PIC assembly to RNA synthesis. Figure 1 depicts the experimental strategy and instrument design. Biotinylated, fluorescently labeled DNA templates containing a consensus Pol II promoter (super core) (Juven-Gershon et al. 2006) were tethered to a biotinylated glass surface via streptavidin. Surface immobilization restricts the DNA Brownian motion and enables long-term tracking of molecular events on each DNA (Fig. 1A). Highly purified human transcription factors (TFIIA, TFIIB, TFIID, TFIIE, TFIIF, TFIIH, and Pol II) (Fig. 1B) and ribonucleoside triphosphates (NTPs) were incubated with the immobilized DNA. Specific interactions of fluorescently labeled factors with the DNA were detected at subsecond time resolution based on spatial colocalization of the point spread functions ("spots") in two fluorescence optical channels (Friedman et al. 2006), thus allowing monitoring of PIC assembly at individual templates. When the transcription reaction was complete, production of RNA from individual templates was detected by imaging of fluorescently labeled DNA oligonucleotide probes.

A typical in vitro Pol II transcription reaction produces $<0.1$ transcript per DNA template per hour (Dignam et al. 1983; Hawley and Roeder 1987; Selby et al. 1997). To detect sufficient numbers of transcription events, we imaged a relatively large $100 \times 100-\mu \mathrm{m}$ field of view and allocated an entire electron-multiplying charge-coupled device (EMCCD) camera sensor per optical channel (Fig. 1C). This allowed monitoring of 1000-3000 discrete DNA templates simultaneously. Active microscope stage stabilization based on real-time image processing was implemented to compensate for sample drift (Fig. 1C, insert) and allow unambiguous, subwavelength colocalization of fluorescent signals throughout the hour-long transcription reactions.
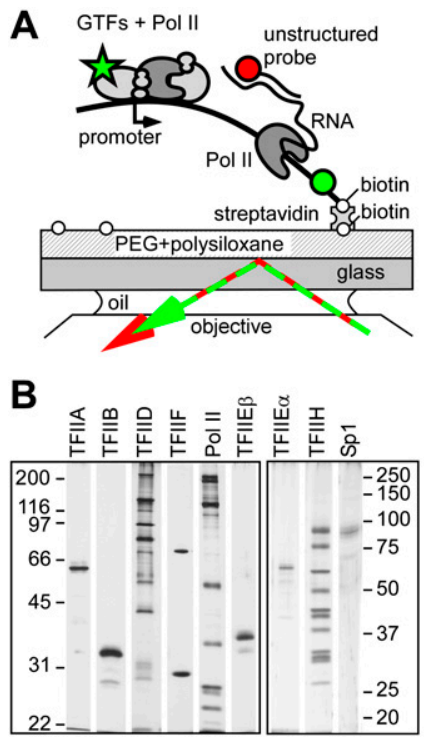

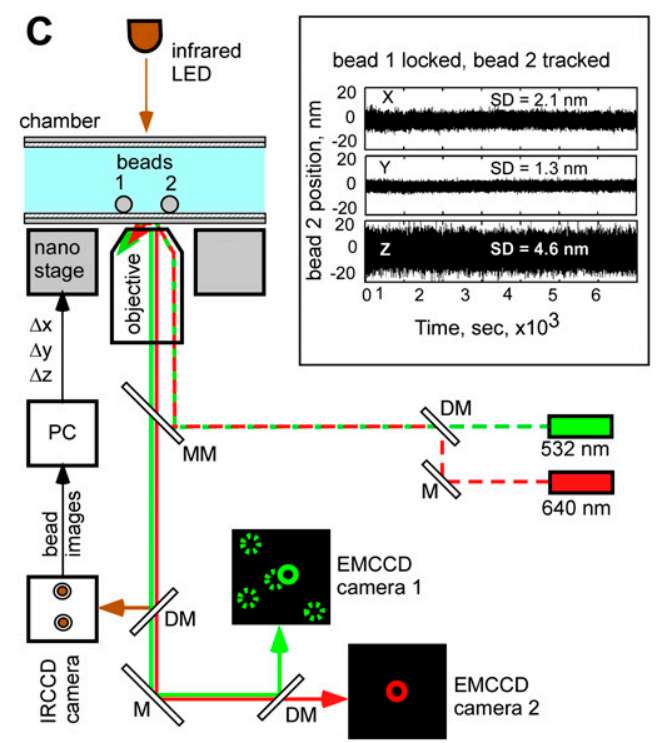

Figure 1. Experimental design and apparatus. (A) Molecular scheme of the experiment. Fluorescently labeled promoter DNA templates are immobilized on a PEGpolysiloxane-coated glass coverslip. A Pol II transcription reaction mixture is supplied, in some cases containing fluorescently labeled TFIID. Transcripts are detected by hybridization of fluorescently labeled unstructured probes. $(B)$ Highly purified protein factors used in the study. Factors were separated by SDS-polyacrylamide gel electrophoresis (PAGE) in $10 \%$ (left) or $6 \%-$ $15 \%$ gradient (right) gels. (C) Imaging setup. A chamber composed of two coverslips is mounted on a three-axis nano-positioning stage (nano stage) of a custom TIRF microscope. Light from 532-nm and 640-nm lasers is combined using mirrors $(\mathrm{M})$ and a dichroic mirror (DM), directed into the microscope using a multichromic mirror (MM), and focused at the periphery of the back focal plane of a 1.49 NA objective to achieve total internal reflection. Fluorescent signal from Cy3/QDot 565 and Cy5 is collected through the same objective and split into two separate optical channels, which are then directed to two separate EMCCD cameras. Spatial colocalization of signals is used as an indication that labeled molecules are associated. For active image stabilization, reference beads are immobilized on the bottom coverslip and illuminated with an infrared LED. The infrared image of beads is diverted from the imaging path using a short-pass DM and detected using an infrared CCD camera. The $(x, y, z)$ position of a bead is determined by real-time image processing (Gosse and Croquette 2002) with a personal computer $(\mathrm{PC})$, and the compensatory displacement information is sent to the nano-positioning stage to maintain the bead at a set point. For simplicity, most optical elements have been omitted. (Insert) Representative $(X, Y$, and $Z)$ time series showing image stabilization. Bead 1 was used as reference, and bead 2 was tracked. Precision as measured by standard deviation (SD) of bead position is indicated for the $X-, Y$-, and $Z$-axes. 
For imaging in our single-molecule setup, Pol II transcription must be initiated on immobilized DNA templates at promoters located within $\sim 100 \mathrm{~nm}$ from the surface (the depth of the evanescent wave in TIRF microscopy), which heightens the risk of protein adsorption and inactivation. The Pol II machinery is complex (>45 polypeptides), and loss of any one component would preclude transcription. We found that commonly used surface passivation methods based on polyethylene glycol (PEG) (Prime and Whitesides 1993; Ha et al. 2002; Kingshott et al. 2002) did not render glass surfaces sufficiently inert to
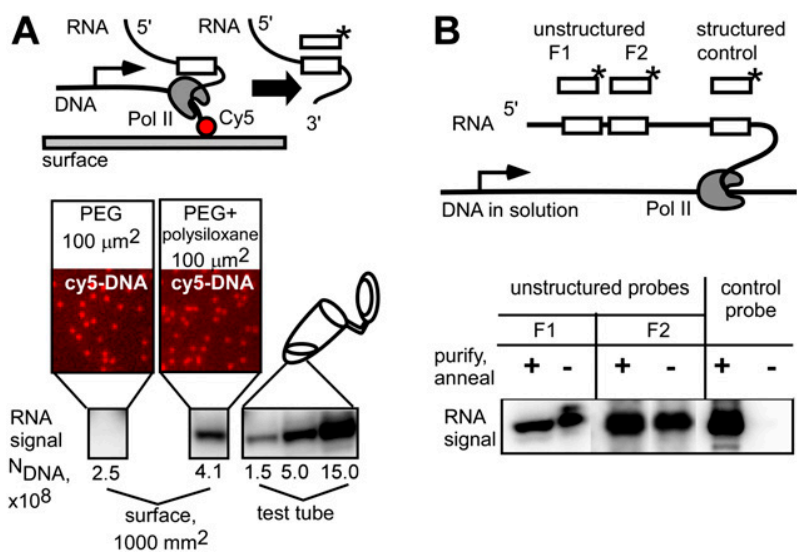

Figure 2. Development of an imaging surface that supports Pol II activity and development of oligonucleotide probes that hybridize to nascent RNA under ambient conditions. (A) Comparison of surface treatments. Cy5-labeled, biotinylated DNA templates containing a consensus Pol II promoter were immobilized on surfaces treated with PEG alone or PEG followed by polysiloxane. DNA density was measured by single-molecule counting. Images show a representative $100-\mu \mathrm{m}^{2}$ region of a $1000-\mathrm{mm}^{2}$ flow cell. The transcription mixture was incubated with the immobilized template, and the RNA product was extracted and annealed to a ${ }^{32} \mathrm{P}$-labeled primer. The rectangular box is the portion of the RNA transcript recognized by a ${ }^{32} \mathrm{P}$-labeled probe. The primer was extended with reverse transcriptase and resolved by urea-PAGE. The bottom panels show primer extension products from the indicated number of immobilized template molecules. As a positive control, transcription was performed using comparable amounts of DNA in solution (test tube). (B) Comparison of conventional and unstructured probes in their ability to detect nascent RNA under ambient conditions. The top panel shows schematic. Open boxes in the transcript indicate the cognate targets for two unstructured probes -F1 and F2 (floppy probes; predicted ensemble free energy of secondary structure, approximately $-0.03 \mathrm{kcal} / \mathrm{mol}$ - and one conventional control probe (same probe as used in $A$; predicted ensemble free energy of secondary structure, approximately $-5 \mathrm{kcal} / \mathrm{mol}$ ). The bottom panel shows primer extension analysis. DNA was transcribed using a Pol II reaction mixture. In reactions labeled "purify, anneal $(+)$, " RNA was purified, and 5 '-radiolabeled probes were annealed to RNA for $1 \mathrm{~h}$ at $55^{\circ} \mathrm{C}$, extended with reverse transcriptase, and resolved by urea-PAGE. In reactions labeled "purify, anneal $(-)$, , purification and separate annealing steps were omitted. Instead, 5 '-radiolabeled probes were present during the transcription reaction to simulate single-molecule transcription conditions, then extended with reverse transcriptase. Representative gel images of the specific extension products are shown. allow Pol II transcription from immobilized DNA templates (Fig. 2A). To address the mechanism of inactivation of transcription activity, we performed biochemical complementation experiments in which transcription mixtures were exposed to PEG-treated glass surfaces, recovered, and supplemented with transcription factors individually or in combination (Supplemental Fig. S1). Results suggested that TFIID (the key GTF comprised of 15 unique subunits and responsible for promoter recognition and activation) (Albright and Tjian 2000) was the most vulnerable to inactivation by the PEG-treated glass surfaces.

We next tried various additional treatments of the glass surface and found that a combination of PEG and 1,7-dichlorooctamethyltetrasiloxane created a much more permissive surface (Fig. 2A). Using the PEG-polysiloxane-treated surface, the efficiency of transcription with glass-immobilized DNA, on a per-template basis, was the same as in conventional solution reactions (Fig. 2A).

Single-molecule imaging of Pol II transcription requires the ability to detect nascent transcripts in near real time (that is, the ability to detect transcripts that remain associated with the single-molecule template of origin). Our approach was based on oligonucleotide probe hybridization. We found that conventional oligonucleotide probes, which hybridize efficiently under conditions in which RNA is purified and annealed for $1 \mathrm{~h}$ at $55^{\circ} \mathrm{C}$, did not hybridize efficiently under conditions that were compatible with maintenance of intact tertiary DNA-Pol II-RNA complexes (Fig. 2B). We hypothesized that a secondary structure in the probe and its RNA target slowed the hybridization reaction. To circumvent this problem, we engineered unstructured ("floppy") probes (predicted ensemble free energy of the secondary structure, $\Delta G_{0} \sim 0.03 \mathrm{kcal} / \mathrm{mol}$ ) (Hofacker 2003). We designed two of these probes (F1 and F2) and inserted their cognate target sequences downstream from the super core promoter. Figure $2 \mathrm{~B}$ shows that, when added directly to the transcription reaction, the floppy probes hybridized efficiently to nascent RNA, as evidenced by the efficiency of primer extension without the additional annealing step. The hybridization was specific because it yielded only one extension product (data not shown).

\section{Single-molecule Pol II transcription detection}

Representative single-molecule transcription data using surface-immobilized templates containing super core promoters are shown in Figure 3. Templates contained a biotin residue and an adjacent $\mathrm{Cy} 3$ or Cy5 fluorophore. The promoter was oriented toward the biotin residue used for DNA immobilization, so that ternary DNAPol II-RNA complexes would mostly arrest when they approach the surface (as evidenced by our bulk transcription experiments showing $\sim 80 \%$ of RNA retained on the surface) (Supplemental Fig. S2). We captured and mapped a few thousand DNA molecules per $100 \times 100-\mu \mathrm{m}$ field. Mapping, based on fitting of the point spread function to a two-dimensional (2D) Gaussian distribution, was with a precision of $<20 \mathrm{~nm}$ (Cheezum et al. 2001; Yildiz and Selvin 2005; Bates et al. 2007). The field was bleached to allow for subsequent reuse of the fluorescent colors. Then 
A

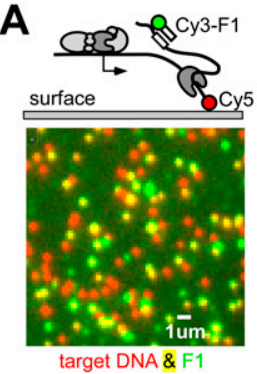

DNA-probe displacement $($ NDNA $=3549)$

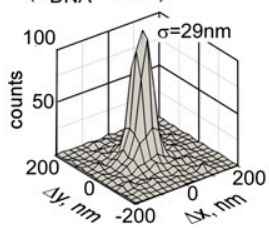

C
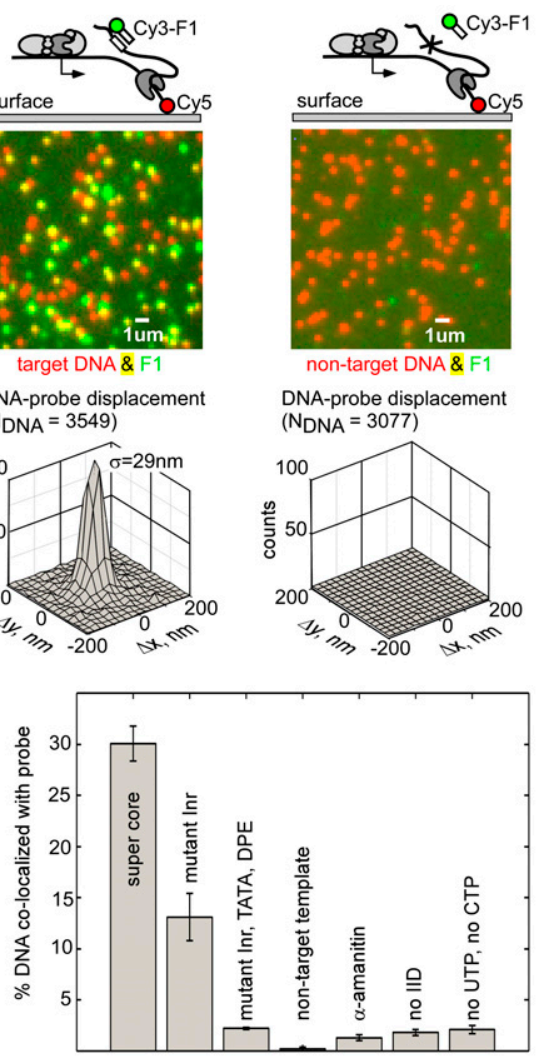

DNA-probe displacement $\left(\mathrm{N}_{\text {DNA }}=3077\right)$

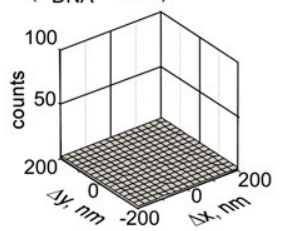

D

D

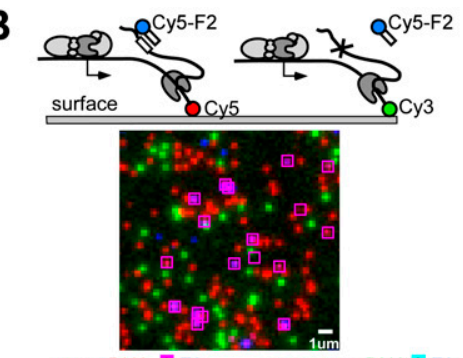

target DNA \& F2 non-target DNA \& F2

DNA-probe displacement DNA-probe displacement
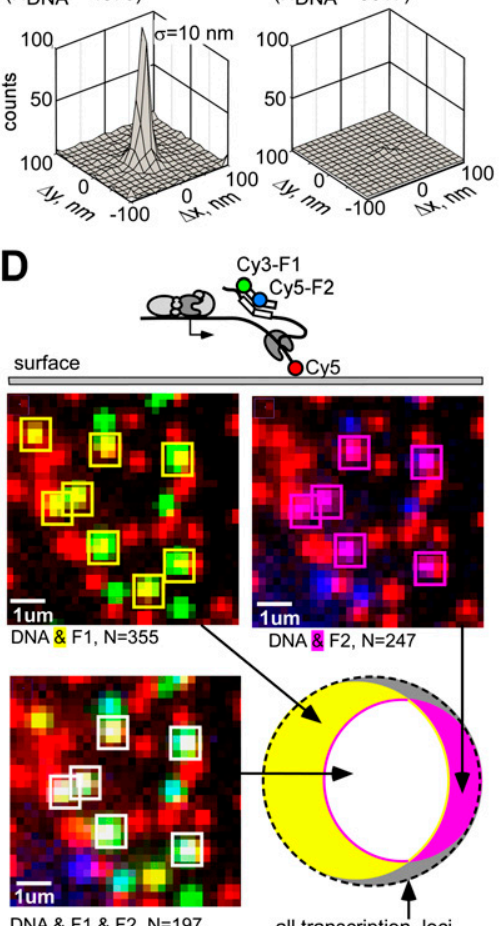

DNA \& F1 \& F2, N=197

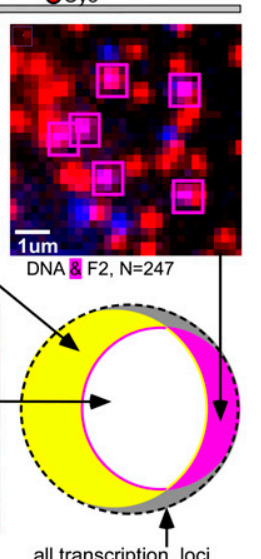

Figure 3. Promoter-specific Pol II transcription measured in the single-molecule assay. (A) Detection of nascent transcripts based on colocalization of floppy probe and template signals. Reactions were performed using Cy5-labeled super core promoter DNA templates containing (left) or lacking (right) the target sequence for the floppy probes. The DNAs were immobilized on a treated glass surface, imaged, and mapped using Gaussian fitting of the point spread function. The fluorophore label was then photobleached. A transcription mixture containing a floppy probe (Cy3-F1) was added and incubated for $45 \mathrm{~min}$ at $30^{\circ} \mathrm{C}$, after which probes were imaged and mapped. Representative $16 \times 16-\mu \mathrm{m}$ regions are shown, with DNA in red and probes in green. Colocalization of DNA and probe signals (appearing as yellow spots) is quantified by 2D DNA-probe displacement histograms using 25-nm binning. (B) Template competition single-molecule assay. Same as $A$, except that target-containing and targetlacking DNA (labeled with Cy5 and Cy3, respectively/ were immobilized in the same flow cell and subjected to detection by the Cy5-labeled F2 probe simultaneously. For display purposes, the F2 probe here was false-colored in blue. Colocalization of target-containing DNA and probe appears as magentas spots (highlighted by magenta squares). Colocalization of target-lacking DNA and probe, if present, would have appeared as cyan spots. $(C)$ Dependence of transcription signal on promoter sequence, target sequence, active Pol II, TFIID, and NTPs. Transcription was measured based on colocalization of DNA and probe signals as in $A$ and $B$ using a three standard deviation cutoff of the DNA probe displacement histogram (Supplemental Fig. S3) and is plotted as a percentage of total DNA in the field of view. Complete reaction using super core promoter is compared with reactions using mutant initiator (Inr) template and a triple mutant template (with mutations in Inr, TATA, and DPE [downstream promoter element]). Other reactions were performed using target-lacking template in the presence of the Pol II inhibitor $\alpha$-amanitin $(0.4 \mathrm{ng} / \mu \mathrm{L})$, in the absence of TFIID, or in the absence of UTP and CTP, as indicated. $(D)$ Efficiency of the unstructured probes in detecting transcribed DNA as determined by simultaneous probing of the same DNA set with two independent probes. The experiment was performed as described in A, except that Cy5-labeled F2 probe was also included in the Pol II transcription reaction mixture. Images show the DNA (red), F1 probe (green), and F2 probe (false-colored blue) merged as indicated. Yellow and magenta squares highlight colocalization of DNA with F1 and F2 signals, respectively, and white squares highlight triple colocalization of DNA, F1, and F2. The Venn diagram summarizes the relationship between populations of DNA templates detected by each individual probe (yellow and magenta ellipses for F1 and F2, respectively) or by both probes simultaneously (intersection of the two ellipses shown in white). The area within the dashed ellipse represents the total predicted number of transcribed loci in the field of view; the gray areas represent the total number of transcription loci that were not detected by either probe. Total $N_{\mathrm{DNA}}=3929$.

GTFs, Pol II, NTPs, and floppy F1 probe were added. Upon incubation to allow RNA synthesis, stalled ternary complexes were washed, RNA probe spots were mapped, and colocalization with the previously mapped DNAs was evaluated. Analytical methods are described in detail in the Supplemental Material.

To evaluate the specificity of probe hybridization under single-molecule conditions, we compared templates containing the probe target sequence with templates lacking the probe target sequence in parallel 45 -min reactions
(Fig. 3A). Overlay images of target-containing DNA and the F1 probe show populations of red spots (Cy5-DNA), green spots (Cy3-F1 probe), and yellow spots (colocalized DNA and probe). The yellow spots are the population of interest; the red and green spots correspond to either unused templates, instances in which DNA colocalized with a probe labeled with a dark Cy3 fluorophore, instances in which a probe colocalized with DNA labeled with a dark Cy5 fluorophore, or nonspecific binding of a probe to the surface. 
In the experiment shown in Figure 3A (left), there are 1059 instances in which the probe signal colocalized within an 87-nm distance of the DNA (of 3549 total DNA spots detected). This suggests an overall template utilization of $30 \%(30.2 \% \pm 1.5 \%$ in experimental repeats). Two lines of evidence argue that the colocalized signal (the yellow spots) reflects specific hybridization of probe to nascent RNA. The first is statistical; the $2 \mathrm{D}$ histogram plot of $(x, y)$ displacements of all DNA-probe colocalization events in Figure 3A shows a strong enrichment at position $(0,0)$ with respect to the DNA /standard deviation $=29 \mathrm{~nm}$ ). Three standard deviations of this histogram thus correspond to the $87-\mathrm{nm}$ threshold used for unbiased DNA-probe colocalization. The estimated colocalization of a randomly positioned probe in such an experiment (generated by offsetting the data sets by $1 \mu \mathrm{m}$ ) was a negligible $0.14 \% \pm 0.05 \%$. The second line of evidence is experimental; no colocalization peak was observed in the parallel reaction using templates containing no target sequence. To further demonstrate the specificity of RNA detection, we performed an experiment in which Cy5-labeled target-containing DNA and Cy3-labeled nontarget DNA were immobilized in the same chamber, imaged, and transcribed simultaneously. Probe colocalization was observed only with the Cy5labeled target-containing DNA (Fig. 3B).

We next performed experiments to evaluate the dependency of transcription on promoter sequence, active Pol II, TFIID, and NTPs. Mutation of the super core promoter, addition of the Pol II inhibitor $\alpha$-amanitin, omission of TFIID, or omission of UTP and CTP significantly or dramatically attenuated the probe-DNA colocalization signal. These results further confirm that the DNA-probe colocalization arises from promoter-specific Pol II transcription (Fig. 3C; Supplemental Fig. S3). The residual $1 \%-2 \%$ nonrandom probe-DNA colocalization in these negative controls was likely due to nonspecific interactions between protein factors, DNA, and the probe.

We then asked whether there was a fraction of transcriptionally active DNA that escaped detection in the floppy probe hybridization assay. The approach was to use two independent floppy probes (F1 and F2) capable of hybridizing to the same transcript. If hybridization is efficient, most transcribed loci will be detected by both probes, whereas if hybridization is inefficient, there will be significant populations of templates that are detected by only one probe or the other. In a representative experiment (Fig. 3D), 197 of 3929 templates showed colocalization with both the F1 and F2 probes, 355 colocalized with F1, and 247 colocalized with F2 (refer to a Venn diagram of DNA populations detected by F1 and/or F2 in Fig. 3D). We draw two conclusions: that detection is not $100 \%$ efficient and that F1 is more efficient than F2. Detection efficiency could not be significantly increased at higher probe concentrations (up to $1 \mu \mathrm{M}$ ) (data not shown), indicating that RNA targets were nearly saturated with the probe. Assuming that hybridization of the F1 and F2 probes to RNA was independent and that RNA hybridization targets were nearly saturated, we can put upper limits on F1 and F2 probe efficiencies. Thus, for probe $\mathrm{F} 1$, the efficiency of detection of transcription sites is the number of transcription sites detected by both F1 and F2 divided by the number of all transcription sites detected by F2 (ratio of the white area to the area of the magenta ellipse in the Venn diagram in Fig. 3D), and thus is equal to 197 of 247 ( $~ 80 \%$, with uncertainties of $\pm 5 \%$ in repeated experiments). Using the same argument, the efficiency of transcription site detection by F2 is $55 \% \pm 5 \%$. As probe labeling efficiency was estimated spectrophotometrically to be near $100 \%$, we suggest that some probe molecules may be nondetectable because the fluorophore is in a dark state (Widengren and Schwille 2000; Huang et al. 2006), a phenomenon that affects Cy5 (F2) more than Cy3 (F1).

Correcting for the $80 \%$ efficiency of active template detection by the F1 probe, the actual template utilization in Figure $3 \mathrm{~A}$ is $37.5 \%(30 \% / 0.8)$, in excellent agreement with the results of bulk biochemistry for the super core promoter (Juven-Gershon et al. 2006). We note that this $80 \%$ probing efficiency by $\mathrm{F} 1$ and $55 \%$ probing efficiency by F2 relate to the probability of detection of transcribed DNA loci and not nascent RNA per se, as some loci are associated with more than one nascent transcript as the result of reinitiation, a phenomenon explored in more detail below. Therefore, the probing efficiency with respect to the transcript would be somewhat lower than the $80 \%$ and $55 \%$ estimates for F1 and F2, respectively.

\section{Counting transcripts at individual DNA templates}

We next sought to determine whether templates that had initiated transcription once were more likely to initiate transcription again. The single-molecule approach is ideal for addressing this question. The quantized nature of the single-molecule fluorescent signals (revealed in single-step fluorescence photobleaching events of the probe) can be used to count the number of probe molecules colocalized with each active DNA and thus determine the number of rounds of transcription that have occurred. Analysis of single-molecule photobleaching steps is used extensively to probe stoichiometry of multisubunit complexes in vivo and in vitro (Shu et al. 2007; Ulbrich and Isacoff 2007; Simonson et al. 2010).

Figure 4A shows three representative probe photobleaching time traces for single-molecule transcription of a super core promoter template containing a single target for the F2 probe. The histogram summarizes data from 223 such traces. About $65 \%$ of the traces showed singlestep photobleaching of the F2 probe, and 35\% showed multistep photobleaching. We interpret the multistep photobleaching as indicative of the presence of multiple RNAs associated with a single template. We excluded the small fraction $(<10 \%)$ of probe traces featuring resolvable $(x, y)$ displacements during consecutive bleaching events (Simonson et al. 2010), which likely originate from two closely spaced DNA templates. DNA photobleached in a single step in $95 \%$ of cases, consistent with the presence of only a single molecule of template at the vast majority of loci. We fit the probability histogram of probe photobleaching steps with a stochastic Poisson model. The Poisson parameter $\lambda$ was $1.1 \pm 0.03$, and the fit was 

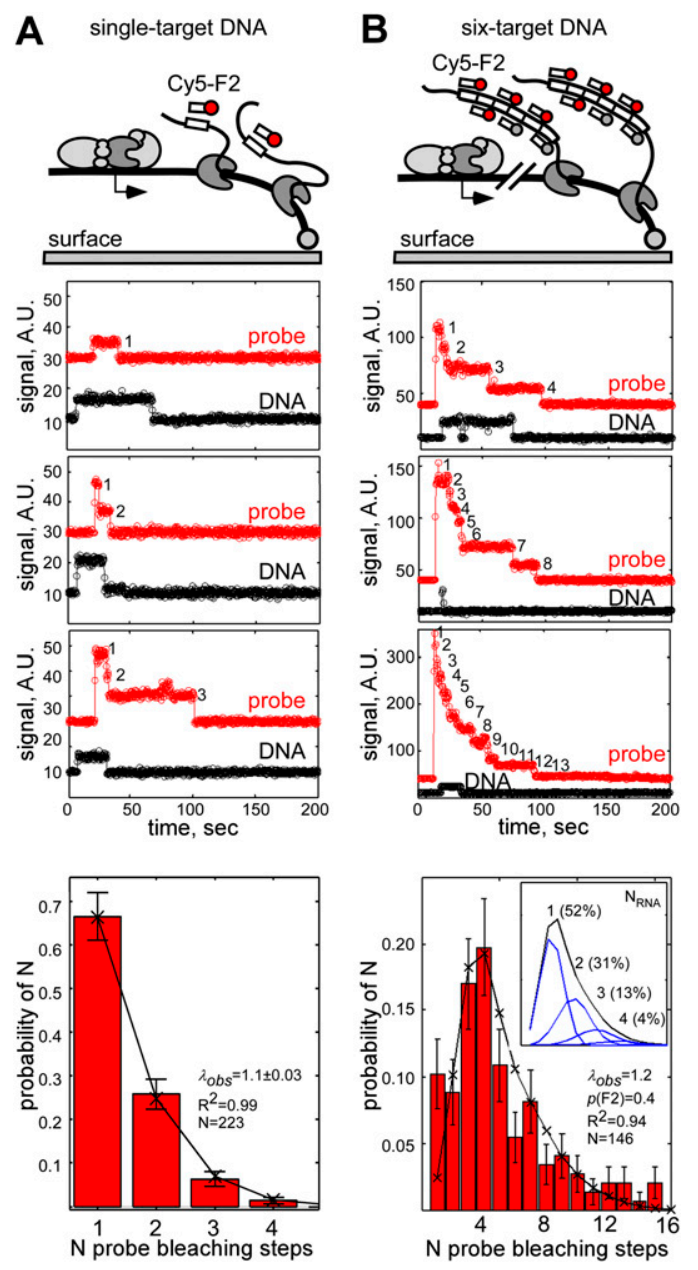

Figure 4. Counting transcripts produced at individual DNA templates. (A) Single-molecule transcription using super core DNA templates containing a single copy of the F2 floppy target. The top panel shows a schematic. The middle panels show representative photobleaching time traces. (Black) Cy5-DNA bleaching traces; (red) Cy5-F2 bleaching traces. The bottom panel shows probability distribution of probe photobleaching steps. Data from $N=223$ transcribed templates were fitted to a zero-truncated Poisson distribution with parameter $\lambda$, with a goodness of fit $\mathrm{R}^{2}=0.99$. (B) Transcription was as in $A$, but with DNA templates containing six copies of the F2 floppy target. Histograms were fit to a combination of a zero-truncated Poisson distribution (with parameter $\lambda$ ) and a sum of binomial distributions of power 6,12, 18, and 24 (with parameter $p$ representing the fraction of nondark Cy5-F2 probes). Relative contributions of templates associated with one, two, three, and four nascent RNAs, calculated based on optimized $\lambda$ and $p$, are shown in the insert for the six-target histogram.

outstanding $\left(R^{2}=0.99\right)$. Of interest, the observed distribution of one, two, three, and four transcription rounds at each DNA was consistent with early well-controlled ensemble experiments that measured the relative contributions of reinitiation rounds by observing polymerases stalled behind a lesion in the DNA template (Szentirmay and Sawadogo 1994).

To reinforce these findings, we performed a second set of experiments using a DNA template containing six tandem repeats of the F2 probe target (Fig. 4B). In an ideal situation (if each RNA was transcribed to completion, and if no dark probes species were present) one would expect to observe maxima at $6,12,18, \ldots, 6 \mathrm{~N}$ steps in the bleaching step probability histogram, corresponding to synthesis of $1,2,3, \ldots, N$ six-target RNAs. The intensity of the maxima would then progressively decrease according to the same Poisson distribution as for the singletarget DNA template (histogram in Fig. 4A). In a real situation, however, the presence of dark probes would blur the maxima and push them toward lower values. Indeed, as shown in Figure 4B, probe bleaching events for the six-target templates were broadly distributed between one and 18 steps, with a maximum at about four steps. We then fit this distribution to a model that assumes the six-target RNAs at each DNA to be stochastically distributed (with the Poisson mean $\lambda$ ) and each of the sixtarget RNA sequences to be randomly sampled by F2 probes containing a fraction $p$ of nondark probes (Fig. 4B; see the Supplemental Material for details). For this model, the mean number of transcripts per DNA for the sixtarget template $\lambda$ was estimated at $\sim 1.2$; the relative contributions of loci containing one, two, three, and four RNAs were estimated at $52 \%, 31 \%, 13 \%$, and $4 \%$, respectively; and the fraction of nondark F2 probes $p$ was estimated at $\sim 0.4\left(R^{2}=0.94\right)$. These findings are in a good agreement with the single-target experiment of Figure 4A. In addition, with these optimized parameters, the expected frequency of colocalization of an active singletarget DNA locus with an F2 probe signal is $\sim 54 \%$ (Supplemental Material), in a good agreement with the independent measurement of $55 \%$ in the two-probe colocalization experiment in Figure 3D.

We also note that saturating amounts of transcription factors were used in all experiments. Thus, the probability of reinitiation or template utilization was unaffected by increasing amounts of TFIID or Pol II threefold or all of the protein factors together 1.5-fold (data not shown), similar to what had been observed in previous bulk studies (Samuels et al. 1982; Sawadogo and Roeder 1985). It is also unlikely that reinitiation was limited by the length of the DNA between the promoter and the glass surface, as this spacer was engineered to accommodate at least six Pol II molecules, assuming the size of the DNA footprint of an elongating Pol II at 40 base pairs (bp) (Selby et al. 1997; Tornaletti et al. 1999).

\section{Activation of single-molecule Pol II transcription by $\operatorname{Sp} 1$}

We next tested whether our single-molecule platform is suitable for studying the regulation of transcription initiation. The prototypical human regulator Spl activates transcription in vitro by recruiting TFIID to the promoter (Dynan and Tjian 1983; Kadonaga et al. 1987; Hoey et al. 1993; Gill et al. 1994). Thus, we introduced a tandem array of three Sp1-binding sites ("GC boxes") upstream of the super core promoter (super core-GC). When tested in a bulk in vitro transcription assay, this DNA template was nonresponsive to Sp1 (Fig. 5A). This result was not 
A

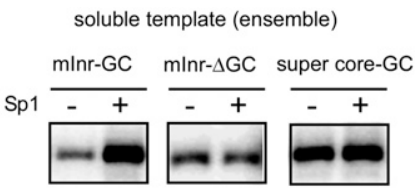

B immobilized template (single-molecule)

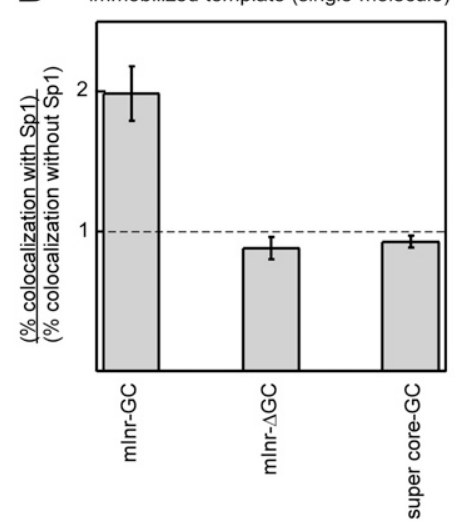

Figure 5. Transcription activation by Sp1. (A) Ensemble reactions using soluble templates. Reactions were performed as described in the Supplemental Material in the presence or absence of $10 \mathrm{nM} \mathrm{Sp1.} \mathrm{Templates} \mathrm{contained} \mathrm{a} \mathrm{super} \mathrm{core}$ promoter with three Spl-binding sites (super core-GC), super core promoter with a mutated initiator element (mInr-GC), or super core promoter with mutated initiator element and lacking the Spl-binding sites (mInr- $\Delta \mathrm{GC})$. Products were detected using a standard primer extension assay. $(B)$ Analogous single-molecule reactions using immobilized templates. Cy3-labeled DNA was transcribed in the presence of Cy5-F2 probe with or without Sp1. The percentage of DNA templates colocalized with probe in the presence of Sp1 was divided by that in the absence of Sp1 and plotted.

entirely unexpected because the super core promoter is optimized for activator-independent TFIID recruitment and might not be further stimulated by an activator that promotes TFIID binding. We thus weakened the super core promoter by introducing a mutation in the Inr element (Smale and Baltimore 1989). In the bulk in vitro assay, the mInr template now displayed a fivefold activation by Sp1. The activation was specific, as it was not seen with a mInr template lacking the Sp1-binding sites (mInr- $\Delta$ GC) (Fig. 5A).

With the single-molecule platform, the mInr template showed a more modest, but reproducible, twofold activation by Sp1 (Fig. 5B). There was no significant change in the distributions of probe photobleaching steps in the presence of Sp1 (data not shown), suggesting that Sp1 did not significantly affect the rate of reinitiation and instead increased template utilization. Mirroring the results of the ensemble assays, no activation by Sp1 was observed with the super core-GC or mInr- $\Delta \mathrm{GC}$ templates.

\section{Correlation of TFIID-DNA interactions with RNA synthesis}

As a first step toward analyzing the roles of individual GTFs in promoter recognition and transcription activity, we devised a strategy to monitor GTF-DNA interactions in real time and correlate these events with productive transcription. As a proof of principle, we used human holo-TFIID labeled at a specific site with a quantum dot. The unique photo-physics of quantum dots makes them particularly suitable for prolonged multicolor imaging, as they have broad excitation spectra, narrow emission spectra, and high photostability (for review, see Michalet et al. 2005). Details of the quantum dot-labeled TFIID (Q-IID) preparation will be published elsewhere. In brief, streptavidin-coated QDot 565 was coupled to a monoclonal antibody specific for the TAF1 subunit of TFIID, which was allowed to interact with native TFIID, after which QDot 565-antibody-TFIID complexes were affinity-purified. Titrations of Q-IID activity showed no difference from native TFIID in a bulk Pol II transcription assay (Supplemental Fig. S4).

We performed single-molecule transcription reactions using Cy5-labeled super core promoter templates. Following DNA photobleaching, we supplied Q-IID instead of the native TFIID in a Pol II transcription reaction mixture and monitored association of Q-IID with the DNA loci in real time at $3 \mathrm{~Hz}$. We counted DNA loci where Q-IID colocalization was observed in at least five frames during the 45-min reaction. Nascent RNAs were detected post-transcriptionally with the Cy5-F2 probe (Fig. 6A).

A representative field from a Q-IID single-molecule transcription experiment is shown in Figure 6B. About $6 \%$ of DNA templates (268 of 4791) were associated with an F2-detectable nascent transcript (magenta squares in Fig. $6 \mathrm{~B}$ ), and $10 \%$ of DNA templates (488 of 4791 ) have interacted with Q-IID during the course of the transcription reaction (yellow squares in Fig. 6B). More importantly, $42 \%$ (113 of 268) of DNA that showed transcription also interacted with Q-IID (white squares in triple-colocalization merged image, see also Venn diagram in Fig. 6B). The overlap between the Q-IID-interacting and transcriptionally active DNA populations was not attributable to chance because a random overlap would correspond to only $\sim 0.6 \%$ of DNA. A major population of templates that interacted with Q-IID did not have a transcript detected (375 of 488 , or $77 \%$ ) (yellow segment in Venn diagram in Fig. 6B). In part, these may be templates where nascent RNA was present but not detected by the F2 probe. This population also includes templates where Q-IID binding was transient or nonproductive.

In a representative time trace showing real-time interactions of Q-IID with a single DNA template (Fig. 6C, top), Q-IID binds within the first $15 \mathrm{~min}$. Statistical analysis of the time intervals between the addition of the transcription reaction mixture $(t=0)$ and arrival of the first Q-IID molecule at the DNA ( $T_{\text {wait }}$ ) revealed a singleexponential probability distribution with the mean lifetime $T_{\text {wait }}=1020 \pm 30 \mathrm{sec}$ (half-life $t_{1 / 2}=T_{\text {wait }} \ln 2=707$ sec) (Fig. 6C, bottom), consistent with previous kinetic measurements in bulk ( $t_{1 / 2}=10-15 \mathrm{~min}$ ) (Hawley and Roeder 1987; Wang et al. 1992; Burley and Roeder 1996) and the notion that TFIID promoter binding is a ratelimiting step of transcription initiation. The mean lifetime measurement for Q-IID binding puts the TFIID on rate on the order of $\sim 10^{6} \mathrm{M}^{-1} \mathrm{sec}^{-1}$ for the super core promoter. 

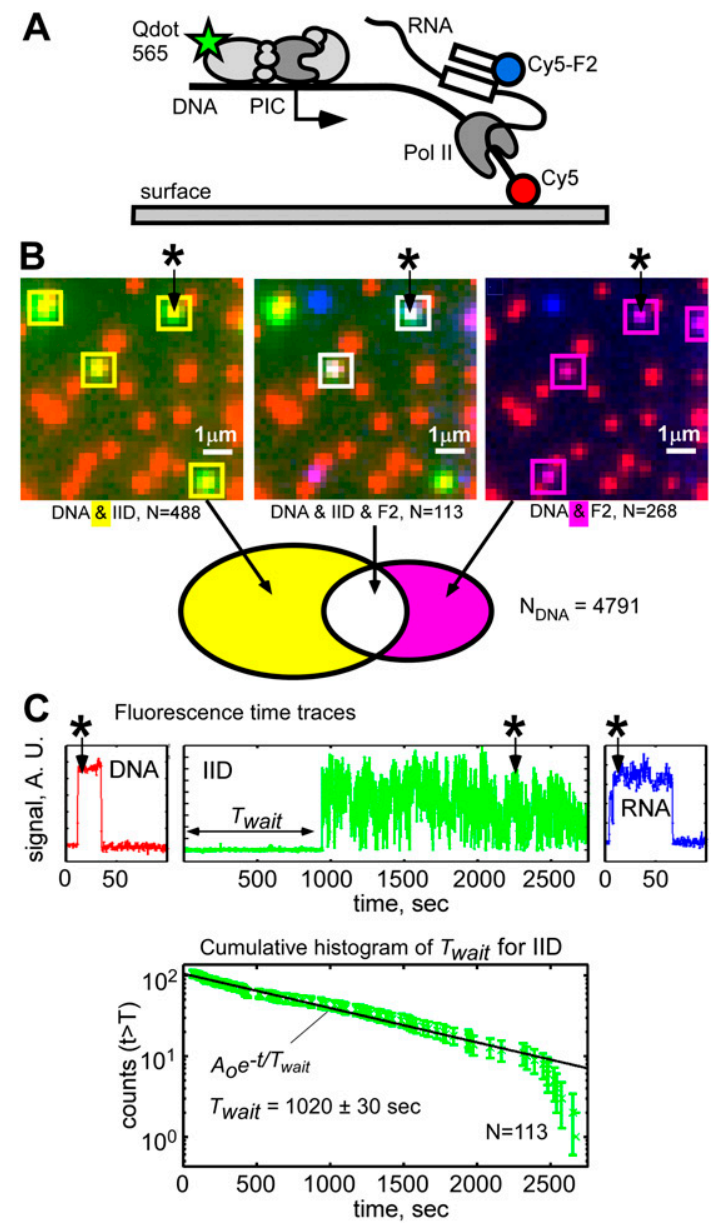

Figure 6. TFIID interactions with individual DNA molecules correlated with transcription. (A) Schematic representation. Cy5-labeled DNA templates were mapped and photobleached, then incubated with complete transcription mixture containing QDot 565-labeled TFIID (Q-IID). Nascent transcripts were detected with Cy5-F2 probes. $(B)$ Representative $8 \times 8-\mu \mathrm{m}$ regions with DNA shown in red, Q-IID in green, and F2 in false-colored blue, merged as indicated. Yellow and magenta squares highlight colocalization of DNA with Q-IID and F2 probe, respectively. White squares indicate triple colocalization of DNA, Q-IID, and F2 probe. Asterisks denote location of the DNA template further analyzed in $C$. The Venn diagram summarizes the relationship between DNA templates associated with Q-IID and templates with transcription detected by the F2 probe. $(C)$ Time trace analysis. Shown at the top are representative time traces of DNA, real-time Q-IID binding, and post-transcriptional detection of RNA by the F2 probe. Shown at the bottom is a cumulative histogram of $T_{\text {wait }}$ of the first Q-IIDbinding events. Asterisks denote time points at which images shown in $B$ were acquired.

The jitter in the Q-IID fluorescent signal reflects the blinking behavior of the quantum dot that is an intrinsic feature of the quantum dot used in this work. The blinking behavior complicates the assignment of the number of times that Q-IID binds and dissociates in the course of the transcription reaction and the measurement of the TFIID off rate. Thus, in this work, we could not unequivocally assign the number of Q-IID-binding events leading to production of a given number of RNAs or determine whether TFIID is part of a "reinitiation scaffold" (Zawel et al. 1995; Yudkovsky et al. 2000). Alternative approaches for preparing covalently labeled TFIID and detection of RNA production in real time may be necessary to address these questions.

\section{Discussion}

Here we report the visualization of promoter-specific transcription initiation in a fully reconstituted Pol II system at the single-molecule level. Due to inherent complexities of this biological system, we had to overcome several critical technological challenges, including multiplexing (i.e., collecting data from thousands of templates simultaneously), active microscope stage stabilization, passivation of the imaging surface, and rapid detection of nascent RNA products. These new technologies, taken in aggregate, should also be suitable for single-molecule reconstruction of complex biochemical reactions, particularly those involving nucleic acid transactions (such as replication, recombination, repair, splicing, and translation).

Multiplexed imaging was critical for detecting Pol II activity due to the well-documented low DNA template usage by Pol II in vitro. Although the model super core promoter used in this work is strong (template usage $~ 40 \%$ ) (Juven-Gershon et al. 2006), native promoters are usually much weaker in vitro (template usage $<5 \%$ ) (Dignam et al. 1983; Hawley and Roeder 1987; Selby et al. 1997). Because single-molecule events are discrete and stochastic, at 5\% template usage, at least 2000 templates need to be interrogated to observe 100 stochastic events with a statistical error of $10 \%(\sqrt{ }(2000 \times 5 \%) / 100=10 \%)$. Therefore, to achieve the required throughput, we chose a fluorescence-based method to assay Pol II initiation, rather than a DNA nanomanipulation technique (Herbert et al. 2008; Lionnet et al. 2012). Studying native promoters transcribed with an even lower efficiency will likely require even higher levels of multiplexing, which can be achieved by stochastic superresolution imaging (Betzig et al. 2006; Rust et al. 2006; Pertsinidis et al. 2010; Berk et al. 2012; Z Zhang and A Revyakin, unpubl.).

Treatment of the imaging surface to prevent adsorption of transcription factors turned out to be a major obstacle. Traditionally, surface quality control in single-molecule studies involves imaging surface interactions of labeled molecules at low-nanomolar concentrations (Selvin and Ha 2008). However, this approach was not practical in our work because $>45$ polypeptides are involved in de novo Pol II initiation. Moreover, PICs tethered to a surface by a 250-bp DNA segment are at a much higher concentration with respect to the surface $(\sim 1 \mu \mathrm{M}$ at one PIC per a hemisphere with a radius of $250 \mathrm{bp} \times 0.34 \mathrm{~nm} / \mathrm{bp}=85$ $\mathrm{nm})$. This high effective concentration, combined with repetitive collisions of PICs with the surface, makes Pol II activity highly susceptible to inactivation. Using a biochemical activity assay that directly measured transcription from immobilized DNA, we systematically tested many alternative surface passivation methods and developed the composite PEG-polysiloxane surface coating. 
However, some residual surface effects on transcription activity cannot be excluded because we found variability in template utilization between batches of passivated glass (e.g., Fig. 3, cf. A and D in terms of template usage). Accordingly, comparative experiments were always performed with the same batch of passivated coverslips.

Another hurdle toward visualizing Pol II transcription at the single-molecule level was the need for rapid and sensitive RNA detection at ambient temperatures. This obstacle was overcome by designing DNA probes and cognate RNA targets that have a low propensity to form secondary structures.

Efficient single-molecule detection of nascent RNA allowed us to count the rounds of transcription at individual promoter DNAs. The probability histogram of transcription rounds fits best with a Poisson model, which is consistent with a noncooperative, independent mechanism of transcription initiation and reinitiation at the super core promoter under our experimental conditions. Our findings therefore do not support previous proposals that a "reinitiation scaffold" of GTFs remains bound at the promoter following Pol II escape and is poised to drive efficient multiple rounds of reinitiation (Zawel et al. 1995; Yudkovsky et al. 2000). Our data indicate that such a scaffold, if present, does not influence the rate-limiting step for initiation at the super core promoter (Kadonaga 1990). Future studies using fluorescently tagged Pol II and GTFs, performed at different promoters, will be required to directly investigate the potential persistence of a reinitiation scaffold after Pol II escape.

A hallmark of transcriptional regulation is the ability of sequence-specific DNA-binding transcription factors, such as the prototypical human regulator Sp1, to enhance initiation by Pol II. Our single-molecule system is indeed responsive to $\mathrm{Sp1}$, with a reproducible twofold effect manifested as an increase in template usage. This result mirrored a fivefold effect of Sp1 observed in a bulk assay. At present, we do not understand why the fold activation by Sp1 was lower in the single-molecule system than in the ensemble reactions, although we cannot rule out the possibility that $\mathrm{Sp} 1$ is adversely affected by interactions with the glass surface. Future single-molecule experiments, involving fluorescently labeled Sp1 and labeled components of the general transcription machinery, combined with real-time RNA detection, will further dissect the mechanism of transcription activation.

Both TFIID and Mediator have been shown to function as coactivators for Sp1-mediated transcription activation in vitro (Pugh and Tjian 1990; Naar et al. 1998; Ryu et al. 1999). However, Mediator did not affect transcription activation in our single-molecule experiments (data not shown). This could be due in part to the use of naked DNA templates and saturating amounts of TFIID in our highly purified system, two conditions known to diminish the requirement for Mediator-dependent activation. Because Mediator dependency is often best observed using chromatin templates (Naar et al. 1998; Taatjes et al. 2002), incorporation of nucleosomes into the DNA templates for single-molecule assays may allow functional studies of Mediator in the future.
Quantum dot-labeled TFIID was used as an initial attempt to correlate PIC assembly and transcription activity at individual DNA templates. The rate of DNA binding by Q-IID agreed well with previous bulk measurements. About half of the transcriptionally active DNA templates displayed prior interaction with Q-IID, while the other half likely represented binding of unlabeled TFIID (potentially due to the dissociation of the fluorescently labeled antibody). Future studies with covalently labeled TFIID should address the issue of label dissociation and will allow a more accurate determination of TFIID dwell times at the promoter DNA and their correlation with productive transcription events.

By directly visualizing DNA templates that interacted with TFIID during transcription, we found that the majority of TFIID-interacting templates $(77 \%)$ did not colocalize with an RNA probe. In part, these may represent templates where nascent RNA was present but not detected. This population may also include templates where PIC assembly was diverted into nonproductive pathways. Indeed, conventional bulk binding assays have suggested that although the occupancy of the promoter DNA by components of the Pol II transcription machinery can be $>50 \%$ (Maldonado et al. 1990; Flores et al. 1991), $<5 \%$ of the templates get transcribed (Dignam et al. 1983). Future single-molecule studies can potentially address the nature of these off-pathway promoter-transcription factor complexes and identify the role they might play in transcription regulation in vivo.

In sum, this study opens the door to new mechanistic insights into the process of PIC assembly, tracking the fates of GTFs during each cycle of transcription and unmasking previously unknown mechanisms of transcriptional activation.

\section{Materials and methods}

\section{Ensemble in vitro transcription}

In vitro transcription and primer extension were based on published protocols (Jones et al. 1985; Pugh and Tjian 1991). TFIIA, TFIIB, TFIIE, and TFIIF were expressed and purified from Escherichia coli, and TFIID, TFIIH, Pol II, and Spl were affinity-purified from HeLa nuclear extracts. Fluorescently labeled, biotinylated DNA templates were PCR-amplified and gel-purified. To monitor the decay of factors due to surface adsorption, a reaction mixture containing GTFs and Pol II (without the DNA template) was incubated between two PEG-treated glass coverslips for $15 \mathrm{~min}$ at $30^{\circ} \mathrm{C}$, recovered, and used to transcribe super core reporter DNA template either directly or after addition of rescue protein factors. For biochemical measurement of transcription activity on surface-immobilized DNA templates, a flow cell with a surface area of $\sim 1000 \mathrm{~mm}^{2}$ was prepared, and DNA molecules were captured, imaged, counted, and transcribed in the flow cell. RNA was recovered and detected by primer extension. The total amount of DNA templates per reaction was obtained by extrapolating the number of DNA molecules counted per field of view $(100 \times 100 \mu \mathrm{m})$ to $1000 \mathrm{~cm}^{2}$. The ability of floppy probes to anneal to nascent RNA during Pol II transcription was examined by directly adding a ${ }^{32} \mathrm{P}$-labeled primer into an in vitro transcription reaction and subjecting the reaction directly to primer extension (without the standard steps of RNA purification and annealing for $1 \mathrm{~h}$ at $55^{\circ} \mathrm{C}$ ). 


\section{Microscope setup}

A custom objective-type TIRF microscope was built around an Olympus IX71 unit equipped with a 1.49 NA $60 \times$ objective and a three-axis nanopositioning stage. Fluorescence of Cy3, QDot 565 , and Cy5 was excited with 532-nm and 640-nm lasers, collected, split into two optical bands (Cy3/QDot 565 and Cy5) and detected with two separate $512 \times 512$-pixel EMCCD cameras (195 nm per pixel). Movies were saved as multiframe TIFF files.

For active image stabilization, beads $\sim 3 \mu \mathrm{m}$ in diameter were captured on the imaging surface and illuminated with an 850-nm LED. Images of beads were detected with a separate CCD camera, the position of a bead in $(x, y, z)$ was determined in real time at $30 \mathrm{~Hz}$ with home-written code and averaged over 30 frames, and compensatory $\Delta x, \Delta y$, and $\Delta z$ were sent to the nanopositioning stage at $1 \mathrm{~Hz}$ for feedback.

\section{Imagining surface preparation}

Glass coverslips were treated with $\gamma$-aminopropyltriethoxysilane and modified with succinimidyl (NHS)-functionalized PEG doped with NHS-PEG-biotin (Ha et al. 2002) in cloud-point conditions (Kingshott et al. 2002). For polysiloxane treatment, PEG-treated coverslips were soaked in $5 \%$ solution of 1,7-dichlorooctamethyltetrasiloxane in heptane for $16 \mathrm{~h}$ at room temperature. After the polysiloxane treatment, the surfaces usually acquire fluorescent background that interferes with imaging of Cy3 and QDot 565 and can be bleached with a 532-nm laser.

\section{Single-molecule transcription}

A multichannel chamber made of two passivated coverslips was mounted on the microscope, and reference beads for stage stabilization were immobilized on the bottom surface of the chamber. Surface was then treated with streptavidin, and Cy3/ Cy5-biotin-labeled DNA molecules were captured. Image stabilization was activated, and DNA templates were imaged at $3 \mathrm{~Hz}$. A Pol II transcription reaction mixture was injected and incubated for $45 \mathrm{~min}$. In most experiments, floppy probes were also present in the reaction mixture, and laser illumination was off during incubation. After incubation, the system was washed, and the floppy probes were imaged. In experiments with Q-IID, laser illumination was on during incubation, and floppy probes were added after transcription, hybridized for $5 \mathrm{~min}$, washed, and imaged.

\section{Single-molecule colocalization analysis}

Fluorescent spots in each movie frame were picked and fit to $2 \mathrm{D}$ Gaussians (Huang et al. 2008) to give subpixel locations of spots in $(x, y)$. A $2 \mathrm{D}$ histogram of spot density in $x$ and $y$ (1 pixel per bin), was built for the entire movie. Adjacent spot-containing bins were merged into clusters. For each cluster in the first movie, a cluster in the second movie located within 1 pixel was found. Displacements between clusters in $x$ and $y$ were plotted as a $2 \mathrm{D}$ histogram $(25-50 \mathrm{~nm}$ per bin) that had a maximum at $(0,0)$ in case of nonrandom colocalization between clusters. The cluster displacement histogram was fitted to a 2D Gaussian to give the standard deviation of displacement, $\sigma$. A cutoff of $3 \sigma$ was used to score colocalization.

\section{Analysis of single-molecule time traces}

For colocalizing spot clusters, time traces were generated for $5-\times-5$-pixel regions of interest, with the central pixel at the rounded location of the colocalizing clusters. Photobleaching step analysis was performed manually using the criteria described in Simonson et al. (2010). Histograms of probe bleaching steps for single-target DNA templates were fit to a zero bintruncated Poisson distribution: $n_{k}=\lambda^{k} e^{-\lambda} / k$ ! (Bancroft et al. 1983). Histograms of probe photobleaching steps for six-target DNA templates were fit to a combination of Poisson and binomial distributions with parameters $\lambda$ (Poisson mean of number of RNA per DNA), and $p$ (the fraction of nondark probes), assuming that up to four transcription rounds occurred, and each RNA of the six-target RNA was transcribed to completion.

See the Supplemental Material for details on experiments and data analysis.

\section{Acknowledgments}

We specially thank William Dynan for help with editing of the manuscript. We thank Yick Fong and Alexey Petrov for comments on the manuscript, Dimitri Chklovskii for suggestions on colocalization analysis, Gleb Shtengel for advice on optics, Bo Huang and Xiaowei Zhuang for spot-finding Insight software, Jonas Korlach and Yu-Chih Tsai (Pacific Biosciences) for discussion and the kind gift of the oxygen-scavenging enzyme, and Crystal Sullivan, Patrice Neville, and Sarah Moorehead for administrative support. A.R. was a Special Fellow of the Leukemia and Lymphoma Society (July 2008-June 2010). Z.Z. was a Fellow of the Leukemia and Lymphoma Society (July 2007June 2009). This work was supported by NIH grant 1P01CA112181-01A1 to S.C. and R.T.

\section{References}

Albright SR, Tjian R. 2000. TAFs revisited: More data reveal new twists and confirm old ideas. Gene 242: 1-13.

Bancroft GA, Colwell DJ, Gilett JR. 1983. A truncated Poisson distribution. Math Gazette 67: 216-218.

Bates M, Huang B, Dempsey GT, Zhuang X. 2007. Multicolor super-resolution imaging with photo-switchable fluorescent probes. Science 317: 1749-1753.

Berk V, Fong JC, Dempsey GT, Develioglu ON, Zhuang X, Liphardt J, Yildiz FH, Chu S. 2012. Molecular architecture and assembly principles of Vibrio cholerae biofilms. Science 337: 236-239.

Betzig E, Patterson GH, Sougrat R, Lindwasser OW, Olenych S, Bonifacino JS, Davidson MW, Lippincott-Schwartz J, Hess HF. 2006. Imaging intracellular fluorescent proteins at nanometer resolution. Science 313: 1642-1645.

Blanchard SC, Kim HD, Gonzalez RL Jr, Puglisi JD, Chu S. 2004. tRNA dynamics on the ribosome during translation. Proc Natl Acad Sci 101: 12893-12898.

Burley SK, Roeder RG. 1996. Biochemistry and structural biology of transcription factor IID (TFIID). Annu Rev Biochem 65: 769-799.

Cheezum MK, Walker WF, Guilford WH. 2001. Quantitative comparison of algorithms for tracking single fluorescent particles. Biophys J 81: 2378-2388.

Dignam JD, Lebovitz RM, Roeder RG. 1983. Accurate transcription initiation by RNA polymerase II in a soluble extract from isolated mammalian nuclei. Nucleic Acids Res 11: 1475-1489.

Dynan WS, Tjian R. 1983. The promoter-specific transcription factor Sp1 binds to upstream sequences in the SV40 early promoter. Cell 35: 79-87.

Flores O, Lu H, Killeen M, Greenblatt J, Burton ZF, Reinberg D. 1991. The small subunit of transcription factor IIF recruits 
RNA polymerase II into the preinitiation complex. Proc Natl Acad Sci 88: 9999-10003.

Friedman LJ, Chung J, Gelles J. 2006. Viewing dynamic assembly of molecular complexes by multi-wavelength singlemolecule fluorescence. Biophys J 91: 1023-1031.

Gill G, Pascal E, Tseng ZH, Tjian R. 1994. A glutamine-rich hydrophobic patch in transcription factor Sp1 contacts the dTAFII110 component of the Drosophila TFIID complex and mediates transcriptional activation. Proc Natl Acad Sci 91: 192-196.

Gosse C, Croquette V. 2002. Magnetic tweezers: Micromanipulation and force measurement at the molecular level. Biophys I 82: 3314-3329.

Ha T, Rasnik I, Cheng W, Babcock HP, Gauss GH, Lohman TM, Chu S. 2002. Initiation and re-initiation of DNA unwinding by the Escherichia coli Rep helicase. Nature 419: 638-641.

Hampsey M. 1998. Molecular genetics of the RNA polymerase II general transcriptional machinery. Microbiol Mol Biol Rev 62: 465-503.

Hawley DK, Roeder RG. 1987. Functional steps in transcription initiation and reinitiation from the major late promoter in a HeLa nuclear extract. I Biol Chem 262: 3452-3461.

Herbert KM, Greenleaf WI, Block SM. 2008. Single-molecule studies of RNA polymerase: Motoring along. Annu Rev Biochem 77: 149-176.

Hoey T, Weinzierl RO, Gill G, Chen JL, Dynlacht BD, Tjian R. 1993. Molecular cloning and functional analysis of Drosophila TAF110 reveal properties expected of coactivators. Cell 72: $247-260$.

Hofacker IL. 2003. Vienna RNA secondary structure server. Nucleic Acids Res 31: 3429-3431.

Hoskins AA, Friedman LJ, Gallagher SS, Crawford DJ, Anderson EG, Wombacher R, Ramirez N, Cornish VW, Gelles J, Moore MJ. 2011. Ordered and dynamic assembly of single spliceosomes. Science 331: 1289-1295.

Huang Z, Ji D, Wang S, Xia A, Koberling F, Patting M, Erdmann R. 2006. Spectral identification of specific photophysics of cy 5 by means of ensemble and single molecule measurements. J Phys Chem A 110: 45-50.

Huang B, Jones SA, Brandenburg B, Zhuang X. 2008. Whole-cell 3D STORM reveals interactions between cellular structures with nanometer-scale resolution. Nat Methods 5: 1047-1052.

Jones KA, Yamamoto KR, Tjian R. 1985. Two distinct transcription factors bind to the HSV thymidine kinase promoter in vitro. Cell 42: 559-572.

Juven-Gershon T, Cheng S, Kadonaga JT. 2006. Rational design of a super core promoter that enhances gene expression. Nat Methods 3: 917-922.

Kadonaga JT. 1990. Assembly and disassembly of the Drosophila RNA polymerase II complex during transcription. I Biol Chem 265: 2624-2631.

Kadonaga JT, Carner KR, Masiarz FR, Tjian R. 1987. Isolation of cDNA encoding transcription factor $\mathrm{Sp} 1$ and functional analysis of the DNA binding domain. Cell 51: 1079-1090.

Kingshott P, Thissen H, Griesser HJ. 2002. Effects of cloud-point grafting, chain length, and density of PEG layers on competitive adsorption of ocular proteins. Biomaterials 23: 2043-2056.

Kornberg RD. 2005. Mediator and the mechanism of transcriptional activation. Trends Biochem Sci 30: 235-239.

Lionnet T, Allemand JF, Revyakin A, Strick TR, Saleh OA, Bensimon D, Croquette V. 2012. Single-molecule studies using magnetic traps. Cold Spring Harb Protoc 2012: 34-49.

Maldonado E, Ha I, Cortes P, Weis L, Reinberg D. 1990. Factors involved in specific transcription by mammalian RNA polymerase II: Role of transcription factors IIA, IID, and IIB during formation of a transcription-competent complex. Mol Cell Biol 10: 6335-6347.

Michalet X, Pinaud FF, Bentolila LA, Tsay JM, Doose S, Li JJ, Sundaresan G, Wu AM, Gambhir SS, Weiss S. 2005. Quantum dots for live cells, in vivo imaging, and diagnostics. Science 307: 538-544.

Naar AM, Beaurang PA, Robinson KM, Oliner JD, Avizonis D, Scheek S, Zwicker J, Kadonaga JT, Tjian R. 1998. Chromatin, TAFs, and a novel multiprotein coactivator are required for synergistic activation by $\mathrm{Sp} 1$ and SREBP-1a in vitro. Genes Dev 12: 3020-3031.

Orphanides G, Lagrange T, Reinberg D. 1996. The general transcription factors of RNA polymerase II. Genes Dev 10: 2657-2683.

Pertsinidis A, Zhang Y, Chu S. 2010. Subnanometre singlemolecule localization, registration and distance measurements. Nature 466: 647-651.

Prime KL, Whitesides GM. 1993. Adsorption of proteins onto surfaces containing end-attached oligo(ethylene oxide): A model system using self-assembled monolayers. I Am Chem Soc 115: 10714-10721.

Pugh BF, Tjian R. 1990. Mechanism of transcriptional activation by Sp1: Evidence for coactivators. Cell 61: 1187-1197.

Pugh BF, Tjian R. 1991. Transcription from a TATA-less promoter requires a multisubunit TFIID complex. Genes Dev 5: 1935-1945.

Roeder RG. 1996. Nuclear RNA polymerases: Role of general initiation factors and cofactors in eukaryotic transcription. Methods Enzymol 273: 165-171.

Rust MJ, Bates M, Zhuang X. 2006. Sub-diffraction-limit imaging by stochastic optical reconstruction microscopy (STORM). Nat Methods 3: 793-795.

Ryu S, Zhou S, Ladurner AG, Tjian R. 1999. The transcriptional cofactor complex CRSP is required for activity of the enhancer-binding protein Sp1. Nature 397: 446-450.

Samuels M, Fire A, Sharp PA. 1982. Separation and characterization of factors mediating accurate transcription by RNA polymerase II. J Biol Chem 257: 14419-14427.

Sawadogo M, Roeder RG. 1985. Factors involved in specific transcription by human RNA polymerase II: Analysis by a rapid and quantitative in vitro assay. Proc Natl Acad Sci 82: 4394-4398.

Selby CP, Drapkin R, Reinberg D, Sancar A. 1997. RNA polymerase II stalled at a thymine dimer: Footprint and effect on excision repair. Nucleic Acids Res 25: 787-793.

Selvin PR, Ha T. 2008. Single-molecule techniques: A laboratory manual. Cold Spring Harbor Laboratory Press, Cold Spring Harbor, NY.

Shu D, Zhang H, Jin J, Guo P. 2007. Counting of six pRNAs of \$29 DNA-packaging motor with customized single-molecule dual-view system. EMBO I 26: 527-537.

Simonson PD, Deberg HA, Ge P, Alexander JK, Jeyifous O, Green WN, Selvin PR. 2010. Counting bungarotoxin binding sites of nicotinic acetylcholine receptors in mammalian cells with high signal/noise ratios. Biophys $J$ 99: L81-L83. doi: 10.1016/j.bpj.2010.08.076.

Smale ST, Baltimore D. 1989. The 'initiator' as a transcription control element. Cell 57: 103-113.

Szentirmay MN, Sawadogo M. 1994. Sarkosyl block of transcription reinitiation by RNA polymerase II as visualized by the colliding polymerases reinitiation assay. Nucleic Acids Res 22: 5341-5346.

Taatjes DJ, Naar AM, Andel F III, Nogales E, Tjian R. 2002. Structure, function, and activator-induced conformations of the CRSP coactivator. Science 295: 1058-1062.

Tornaletti S, Reines D, Hanawalt PC. 1999. Structural characterization of RNA polymerase II complexes arrested by 


\section{Revyakin et al.}

a cyclobutane pyrimidine dimer in the transcribed strand of template DNA. I Biol Chem 274: 24124-24130.

Uemura S, Aitken CE, Korlach J, Flusberg BA, Turner SW, Puglisi ID. 2010. Real-time tRNA transit on single translating ribosomes at codon resolution. Nature 464: 1012-1017.

Ulbrich MH, Isacoff EY. 2007. Subunit counting in membranebound proteins. Nat Methods 4: 319-321.

Wang W, Gralla JD, Carey M. 1992. The acidic activator GAL4$\mathrm{AH}$ can stimulate polymerase II transcription by promoting assembly of a closed complex requiring TFIID and TFIIA. Genes Dev 6: 1716-1727.

Weiss S. 1999. Fluorescence spectroscopy of single biomolecules. Science 283: 1676-1683.

Widengren J, Schwille P. 2000. Characterization of photoinduced isomerization and back-isomerization of the cyanine dye Cy 5 by fluorescence correlation spectroscopy. I Phys Chem A 104: 6416-6428.

Yildiz A, Selvin PR. 2005. Fluorescence imaging with one nanometer accuracy: Application to molecular motors. Acc Chem Res 38: 574-582.

Yudkovsky N, Ranish JA, Hahn S. 2000. A transcription reinitiation intermediate that is stabilized by activator. Nature 408: 225-229.

Zawel L, Kumar KP, Reinberg D. 1995. Recycling of the general transcription factors during RNA polymerase II transcription. Genes Dev 9: 1479-1490. 


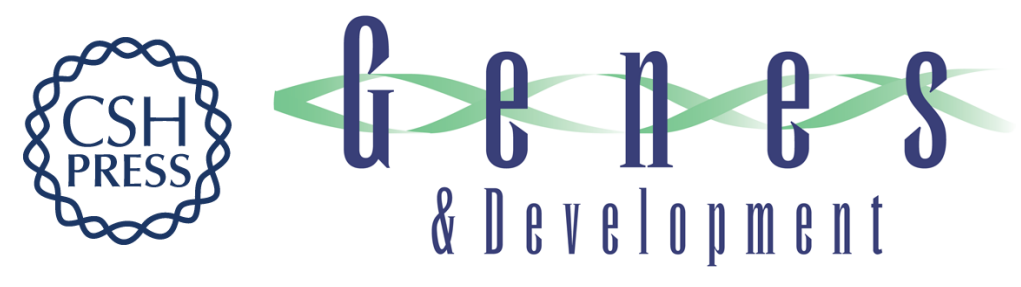

\section{Transcription initiation by human RNA polymerase II visualized at single-molecule resolution}

Andrey Revyakin, Zhengjian Zhang, Robert A. Coleman, et al.

Genes Dev. 2012, 26: originally published online July 18, 2012

Access the most recent version at doi:10.1101/gad.194936.112

\section{Supplemental http://genesdev.cshlp.org/content/suppl/2012/07/12/gad.194936.112.DC2 \\ Material}

Related Content Eeny meeny miny moe, catch a transcript by the toe, or how to enumerate eukaryotic transcripts

Terence R. Strick and Nouria Hernandez

Genes Dev. August , 2012 26: 1643-1647

References This article cites 58 articles, 22 of which can be accessed free at: http://genesdev.cshlp.org/content/26/15/1691.full.html\#ref-list-1

Articles cited in:

http://genesdev.cshlp.org/content/26/15/1691.full.html\#related-urls

\section{License}

Email Alerting

Service

Receive free email alerts when new articles cite this article - sign up in the box at the top right corner of the article or click here.

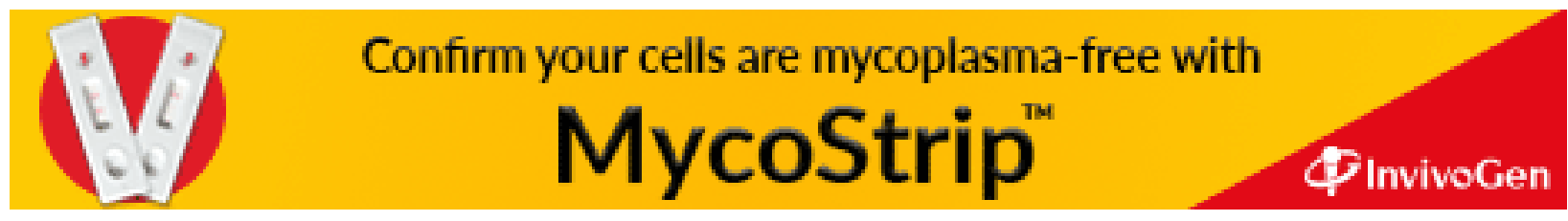

